\title{
Superconductivity and Magnetism in Pure and Substituted $\operatorname{RuSr}_{2} \mathrm{GdCu}_{2} \mathbf{O}_{8}{ }^{1}$
}

\author{
B. Dabrowski, ${ }^{2}$ P. W. Klamut, ${ }^{2}$ M. Maxwell, ${ }^{2}$ S. M. Mini, ${ }^{2}$ S. Kolesnik, ${ }^{2}$ \\ J. Mais, ${ }^{2}$ A. Shengelaya, ${ }^{3,4}$ R. Khazanov, ${ }^{3,4}$ H. Keller, ${ }^{3,4}$ C. Sulkowski, ${ }^{5}$ \\ D. Wlosewicz, ${ }^{5}$ and M. Matusiak ${ }^{5}$
}

We describe results of several macroscopic and local measurements of magnetic and super-
conducting properties for pure and $\mathrm{Ce}$ and $\mathrm{Cu}$ substituted $\mathrm{RuSr}_{2} \mathrm{GdCu}_{2} \mathrm{O}_{8}$. From various
experiments the physical properties phase diagram is derived as a function of the hole doping.
We show that the large contribution of polarized $\mathrm{Gd}^{3+}$ magnetic moments to magnetization
causes apparent absence of the Meissner state and augments the weak Ru ferromagnetism.
This weak ferromagnetism in the superconducting state can be significantly enhanced by the
external magnetic field. These results indicate that the low-temperature behavior can be quali-
tatively explained assuming a quasi-two-dimensional character of the superconducting regions.

KEY WORDS: superconductivity; magnetism; ruthenocuprates; coexistence.

\section{INTRODUCTION}

Owing to their chemical versatility and structural intricacy, complex oxides attain several unique electronic and magnetic properties that can be creatively altered by design of crystal chemistry. During the past 15 years a wide variety of copper-oxide hightemperature superconductors [1] (HTSC) have been discovered and optimized for increased superconducting transition temperature, irreversibility fields, and critical currents. Recently, an apparent coexistence of superconductivity (SC) and ferromagnetism was reported in $\mathrm{RuSr}_{2} \mathrm{GdCu}_{2} \mathrm{O}_{8}$ ( $\left.\mathrm{Ru} 1212\right)[2,3] \mathrm{com}-$ pound at unusually high $T_{\mathrm{c}}=45$ and $T_{\mathrm{m}}=132 \mathrm{~K}$, respectively. The unique properties have stimulated intense interest in this material.

\footnotetext{
${ }^{1}$ This paper was presented at the SCRM-2002 Conference, Gienes, France, June 1-8, 2002.

${ }^{2}$ Department of Physics, Northern Illinois University, DeKalb, Illinois 60115.

${ }^{3}$ Physik-Institut der Universitaet Zuerich, CH-8057, Villigen, Switzerland.

${ }^{4}$ Paul Sherrer Institute, Villigen, Switzerland.

${ }^{5}$ Institute of Low Temperature and Structure Research of Polish Academy of Sciences, 50-950 Wroclaw, Poland.
}

Ginzburg [4] and Matthias [5] have discussed coexistence of SC and ferromagnetism and determined that the spin-singlet SC and ferromagnetism are incompatible. However, microscopic coexistence was discovered in certain low-temperature f-electron superconductors [6] suggesting unconventional (nonsinglet) pairing states and this area of research is still very active. Several heavy-fermion superconductors exhibit coexistence of weak antiferromagnetism $(\mathrm{AFM})$ and $\mathrm{SC}$ with $T_{\mathrm{N}}>T_{\mathrm{c}}$. $\mathrm{URu}_{2-x} \mathrm{M}_{x} \mathrm{Si}_{2}(\mathrm{M}=\mathrm{Re}$, Tc) was the first example of a compound exhibiting ferromagnetic instability in a heavy electron system [7]. Recently, SC was shown to exist to the lowest temperatures within the ferromagnetic state for an itinerant electron system $\mathrm{UGe}_{2}$ [8], suggesting the association of SC with ferromagnetic spin fluctuations, i.e., magnetic spin-triplet origin of the Cooper pairing.

The complex crystal structure of HTSC illustrated schematically on Fig. 1 allows changing their properties by control of the atomic arrangements. $\mathrm{Ru} 1212$ is a layered triple perovskite similar to Y123 with $\mathrm{Ba}^{2+}$ and $\mathrm{Y}^{3+}$ replaced by $\mathrm{Sr}^{2+}$ and $\mathrm{Gd}^{3+}$, respectively, and containing $\mathrm{RuO}_{2}$ planes instead of the $\mathrm{CuO}$ chains in the blocking region of the structure. Under normal synthesis conditions this special layered order 


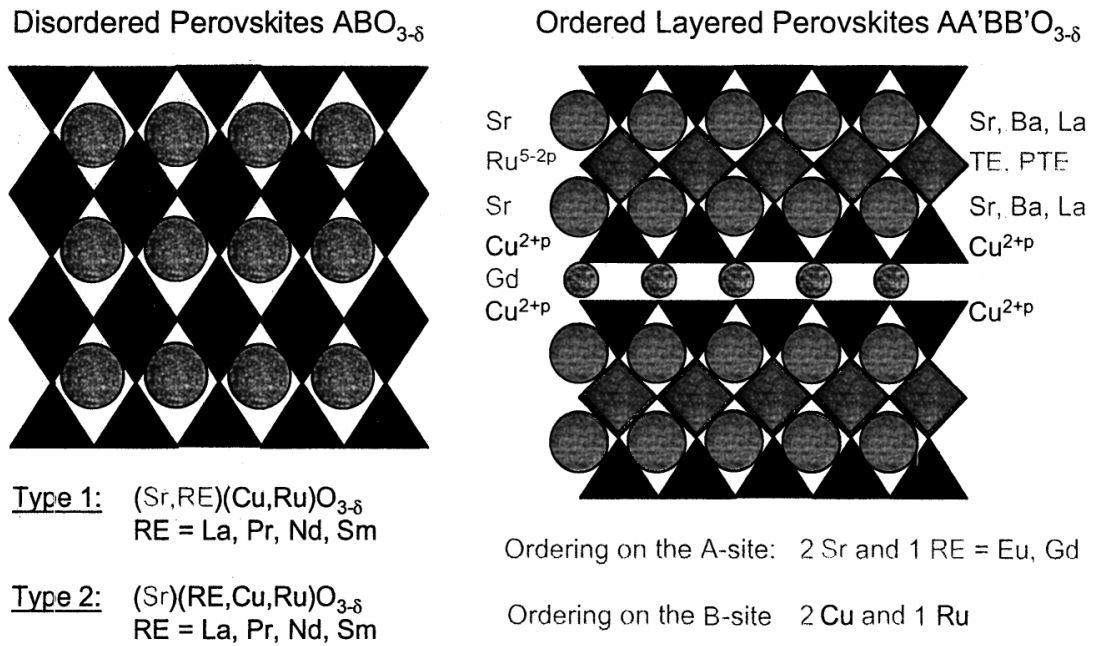

Fig. 1. Schematic structure of the disordered on the A- and B-sites (left panel) and layered ordered (right panel) perovskites.

of both large $\left(\mathrm{A}=\mathrm{Sr}, \mathrm{A}^{\prime}=\right.$ Rare Earth's $)$ and small $\left(\mathrm{B}=\mathrm{Cu}, \mathrm{B}^{\prime}=\mathrm{Ru}\right)$ cations can be accomplished only for $\mathrm{A}^{\prime}=\mathrm{Eu}$ and $\mathrm{Gd}$. For larger Rare Earth's, $\mathrm{A}^{\prime}=$ $\mathrm{La}-\mathrm{Sm}$, mixed perovskites are formed with randomly distributed $\mathrm{Sr}$ and Rare Earth's on the A-site, and $\mathrm{Cu}$ and $\mathrm{Ru}$ on the B-site. For smaller Rare Earth's, $\mathrm{A}^{\prime}=\mathrm{Dy}-\mathrm{Lu}$, another mixed perovskites are formed with the Rare Earth's, $\mathrm{Cu}$, and $\mathrm{Ru}$ occupying the B-site.

Muon spin rotation and magnetization measurements provided evidence for the coexistence of magnetic ordering of Ru moments with SC (see Ref. 2). Although ferromagnetic ordering was initially proposed for the Ru sublattice [2] recent neutron diffraction experiments in agreement with band structure computations [9] show that the Ru moments form the G-type AFM structure with magnetic moments perpendicular to the c-axis [10-12]. The observed weak ferromagnetism, clearly indicated by hysteresis loops and temperature irreversibility of magnetization, might originate in the $\mathrm{RuO}_{2}$ planes from canting of the AFM lattice and may result in the effective field being parallel to the $\mathrm{CuO}_{2}$ planes [12,13]. According to the first principle calculations by Pickett et al. [13], Ru1212 can be approximated by a quasi-two-dimensional system in the magnetic fields nearly parallel to the SC layers. It has also been suggested that Ru1212 is a candidate compound for the occurrence of a spatially modulated superconducting order parameter [14]. According to Ref. 14, the complex structure of the Fermi surface allows for coexistence of ferromagnetism and SC on a microscopic scale by enhancing the critical field of the SC layer. Substantially modified properties of the vortex lattice and the absence of the Meissner state were among the unusual properties proposed for these compounds [15].

Using high-oxygen-pressure and "in situ" thermogravimetric synthesis, we have found that it is possible to prepare a wide range of substituted Ru1212 compounds with atomically ordered structures by controlling the chemical composition, temperature, and oxygen partial pressure. By optimizing the synthesis conditions, we have significantly extended the range of solubility limits of $\mathrm{Ce}^{4+}$ substitution for $\mathrm{Gd}$ and discovered new phases with $\mathrm{Cu}$ substituted for $\mathrm{Ru}$. Increased magnetic and superconducting transition temperatures have been observed for these materials. We have studied the correlated structureproperty phase diagrams of these compounds by combining the results of macroscopic dc magnetization, ac susceptibility, resistivity, thermopower, Hall effect, specific heat, and structural neutron and X-ray powder diffraction measurements performed as a function of temperature and applied magnetic field. In addition, we have used muon spin rotation experiments, Mossbauer spectroscopy, and synchrotron Xray Absorption Near Edge Spectroscopy (XANES), to examine local properties, atomic arrangements, and valence states of $\mathrm{Ru}$ and $\mathrm{Cu}$. Here we describe briefly the main results of these measurements that suggest that the low-temperature behavior can be qualitatively explained assuming a quasitwo-dimensional character of the superconducting regions [16]. 


\section{EXPERIMENTAL PROCEDURE}

Polycrystalline samples of pure and substituted $\mathrm{RuSr}_{2} \mathrm{GdCu}_{2} \mathrm{O}_{8}$ were prepared by the solid-state reaction from a stoichiometric mixture of oxides of $\mathrm{Cu}$, $\mathrm{Ru}, \mathrm{Gd}$, and $\mathrm{Ce}\left(\mathrm{RuO}_{2}\right.$ was prefired in oxygen at $800^{\circ} \mathrm{C}$ and carefully handled before weighing to avoid formation of hydroxides) and carbonates of $\mathrm{Sr}$ and $\mathrm{Ca}$. Samples were fired under controlled environment for several days with frequent intermediate grindings, checked for phase purity, annealed under several oxygen pressures and temperatures, and checked for $T_{\mathrm{c}}$ and $T_{\mathrm{m}}$. The high pressure anneals were done for $12 \mathrm{~h}$ in $20 \% \mathrm{O}_{2}$ in argon at a total pressure of $3 \mathrm{kbar}(600$ atm. $\mathrm{O}_{2}$ pressure) at $1050-1120^{\circ} \mathrm{C}$ followed by slow cooling to room temperature. The crystal structure and lattice parameters were determined by powder $\mathrm{X}$-ray diffraction for $\mathrm{Ce}, \mathrm{Ca}$, and $\mathrm{Cu}$ substituted materials. Neutron powder diffraction data were obtained using the Spatial Environment powder Diffractometer at Argonne National Laboratory Intense Pulsed Neutron Source for pure Ru1212 samples substituted with ${ }^{150} \mathrm{Gd}$ and ${ }^{153} \mathrm{Eu}$. Susceptibility and magnetization measurements were performed with a Quantum Design PPMS system. Resistivity was measured using a standard four-lead dc method. The Mossbauer ${ }^{155} \mathrm{Gd}$ and ${ }^{99} \mathrm{Ru}$ effect measurements were performed using a standard constant accelerator spectrometer. The XANES measurements were carried out at the ChemMat CARS undulators beam line at the Advanced Phonon Source in Argonne. Zero-field and transverse-field muon spin rotation experiments were performed at Paul Sherrer Institute in Villigen.

\section{SUBSTITUTIONS AND SYNTHESIS}

The Sr-based 123 compounds have been investigated extensively for more than a decade [17]. It was found from these studies that certain transition and posttransition metals ( $\mathrm{Fe}, \mathrm{Co}, \mathrm{Al}$, and $\mathrm{Ga}$ ) are capable to completely replace copper in the chain region of the structure resulting in nonsuperconducting materials. These elements and several others (Ti, V, W, Mo, Re) can form also an extensive solubility with $\mathrm{Cu}$ in a range from 0 up to $85 \%$. Solid solutions of the lower valence $\mathrm{Cu}$ substituting for the higher valence transition metals usually resulted in an increased hole-doping of the $\mathrm{CuO}_{2}$ planes and the appearance of superconductivity near the solubility limit. These known facts suggested to us that it should be possible to change the doping level of the $\mathrm{CuO}_{2}$ planes in $\mathrm{RuSr}_{2} \mathrm{GdCu}_{2} \mathrm{O}_{8}$ and to modify the magnetic and superconducting properties by substitutions.

We have investigated the effects of variety of synthesis conditions on the sample quality and properties [18]. Samples synthesized in $1 \%$ oxygen/Ar at $930^{\circ} \mathrm{C}$ were nonsuperconducting for pure Ru1212. Prolonged annealing in oxygen at $1060^{\circ} \mathrm{C}$ gradually induced superconductivity. Simultaneously, the magnetic transition was slightly shifted to lower temperatures. The thermogravimetric analysis could not detect observable changes in oxygen content among various samples. This indicates that the effects other than the average oxygen doping, for example, the degree of the layered ordering or the cation disorder, may affect $T_{\mathrm{c}}$ and $T_{\mathrm{m}}$ for pure material.

To change the charge doping, we have attempted two kinds of substitutions: the heterovalent $\mathrm{Ce}^{4+}$ and $\mathrm{Ca}^{2+}$ for $\mathrm{Gd}$ that leaves both $\mathrm{Ru}-\mathrm{O}$ and $\mathrm{Cu}-\mathrm{O}$ planes intact, and $\mathrm{Cu}$ for $\mathrm{Ru}$ that dilutes the magnetic sublattice of Ru. Single-phase samples of $\mathrm{RuSr}_{2} \mathrm{Gd}_{1-y} \mathrm{Ce}_{y} \mathrm{Cu}_{2} \mathrm{O}_{8}$ with $y=0-0.1$ have been successfully synthesized [19] in oxygen at $1060^{\circ} \mathrm{C}$ and in $1 \%$ oxygen $/ \mathrm{Ar}$ at $930^{\circ} \mathrm{C}$. The Ca substituted mate$\operatorname{rial}(y=0.1)$ synthesized by the same procedures had clearly increased SC transition (to $61 \mathrm{~K}$ ) indicating increased hole-doping; however, we were not able to find synthesis conditions for preparation of the singlephase material (see Fig. 2).

Samples of $\mathrm{Ru}_{1-x} \mathrm{Sr}_{2} \mathrm{GdCu}_{2+x} \mathrm{O}_{8-d}(x=0-0.75)$ could be only prepared at high-pressure oxygen atmosphere at $1050-1120^{\circ} \mathrm{C}$. Figure 2 shows an example of the X-ray pattern for the sample $(x=0.5)$ prepared in air, annealed at high oxygen pressure, and fired again in air without intermediate grindings. The Ru1212 phase prepared in 600 bar at $1065^{\circ} \mathrm{C}$ decomposes in air. Repeated annealing under high-pressure oxygen conditions with intermediate grinding improves the phase purity of the material without appreciable change of the superconducting and magnetic properties. Smooth decrease of the tetragonal lattice parameters $a$ and $b$ confirmed that considerably increased hole-doping can be achieved with the $\mathrm{Cu}$ substitution and an increased electron doping with the $\mathrm{Ce}$ substitution.

\section{COUPLED SUPERCONDUCTIVITY AND MAGNETISM}

ac susceptibility and dc magnetization measurements for solid chunks and powders of pure Ru1212 clearly showed presence of magnetic transition at $T_{\mathrm{m}}=132 \mathrm{~K}$ and SC transition at lower temperatures. 


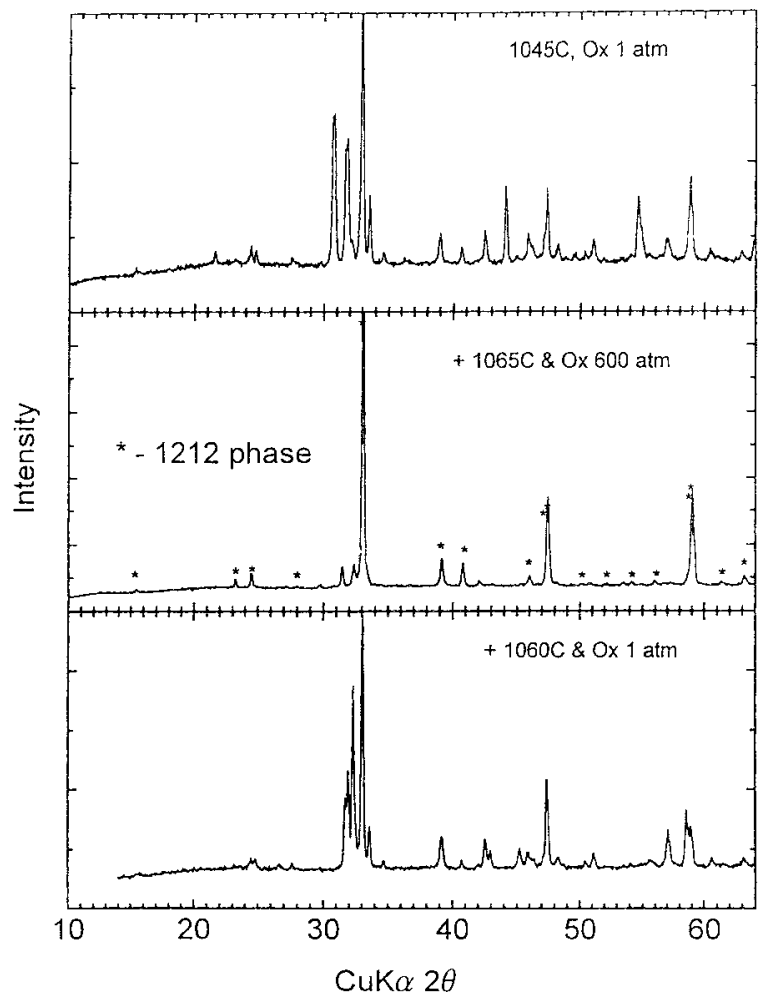

Fig. 2. X-ray diffraction patterns for the $\mathrm{Ru}_{0.5} \mathrm{Sr}_{2} \mathrm{GdCu}_{2.5} \mathrm{O}_{8-d}$ samples prepared in air at $1045^{\circ} \mathrm{C}$ (top), in $600 \mathrm{~atm} . \mathrm{O}_{2}$ at $1065^{\circ} \mathrm{C}$ (middle), and annealed in air at $1060^{\circ} \mathrm{C}$ without intermediate grindings (bottom). Presence of the small amount of second phase is still visible for high pressure $\mathrm{O}_{2}$ annealed sample.

The SC transition always revealed two characteristic features: the onset of transition at $T_{\mathrm{c} 1} \sim 45 \mathrm{~K}$ and the establishment of the bulk superconducting screening currents at $T_{\mathrm{c} 2} \sim 35 \mathrm{~K}$. Comparison of the $\mathrm{M}(\mathrm{H})$ curves at $4.5 \mathrm{~K}$ for superconducting $\mathrm{RuSr}_{2} \mathrm{GdCu}_{2} \mathrm{O}_{8}$ with nonsuperconducting $\mathrm{GdBa}_{2} \mathrm{Cu}_{3} \mathrm{O}_{62}$ unveiled dominant contribution of the polarized $\mathrm{Gd}^{3+}$ magnetic moments to the magnetization, and the presence of an additional contribution developing with increasing magnetic field. After subtracting contribution of polarized $\mathrm{Gd}^{3+}$ magnetic moments the additional increment rising to $\sim 1 \mu_{\mathrm{B}}$ per formula at $H_{\mathrm{dc}}=6 \mathrm{~T}$ was identified as the ferromagnetic alignment of the $\mathrm{Ru}$ moments. This ferromagnetic alignment is observed for values of the magnetic field that are below the critical field for the superconducting phase (see Ref. 16), i.e., weak Ru ferromagnetism observed for superconducting Ru1212 is significantly enhanced in the presence of the magnetic field. Comparison between the properties of the $\mathrm{Gd}^{3+}\left(M_{\mathrm{eff}} \approx 7.94 \mu_{\mathrm{B}}\right)$ and the nonmagnetic $\mathrm{Eu}^{3+}$ based compounds showed that polarized $\mathrm{Gd}^{3+}$ magnetic moment enhances also the weak ferromagnetism of the $\mathrm{Ru}$ sublattice. Large paramagnetic contribution of $\mathrm{Gd}^{3+}$ leads to reentrant behavior of magnetization at low temperatures causing apparent absence of the Meissner state. This behavior can be visualized by inferring quasi-two-dimensional character of the SC layers that are separated by nonsuperconducting regions. For polycrystalline samples with randomly oriented crystallites, the paramagnetic response would arise from the crystallites for which SC layers are oriented parallel to the external field that can penetrate the nonsuperconducting regions between them (see Fig. 3).

The Ce substitution (electron doping) lowers $T_{\mathrm{c} 1}$ from $45 \mathrm{~K}$ for $y=0-36 \mathrm{~K}$ for $y=0.02 ; y=0.05$ and 0.1 compositions are nonsuperconducting (see Fig. 3 (top panel)). The temperature of magnetic ordering increases almost linearly from 132 to $190 \mathrm{~K}$ for $y=0$ and 0.1 , respectively. The onset temperatures for $\mathrm{Cu}$ substituted samples increase from $T_{\mathrm{c} 1}=45 \mathrm{~K}$ for $x=0-72 \mathrm{~K}$ for $x=0.3$ and 0.4 , and then decrease

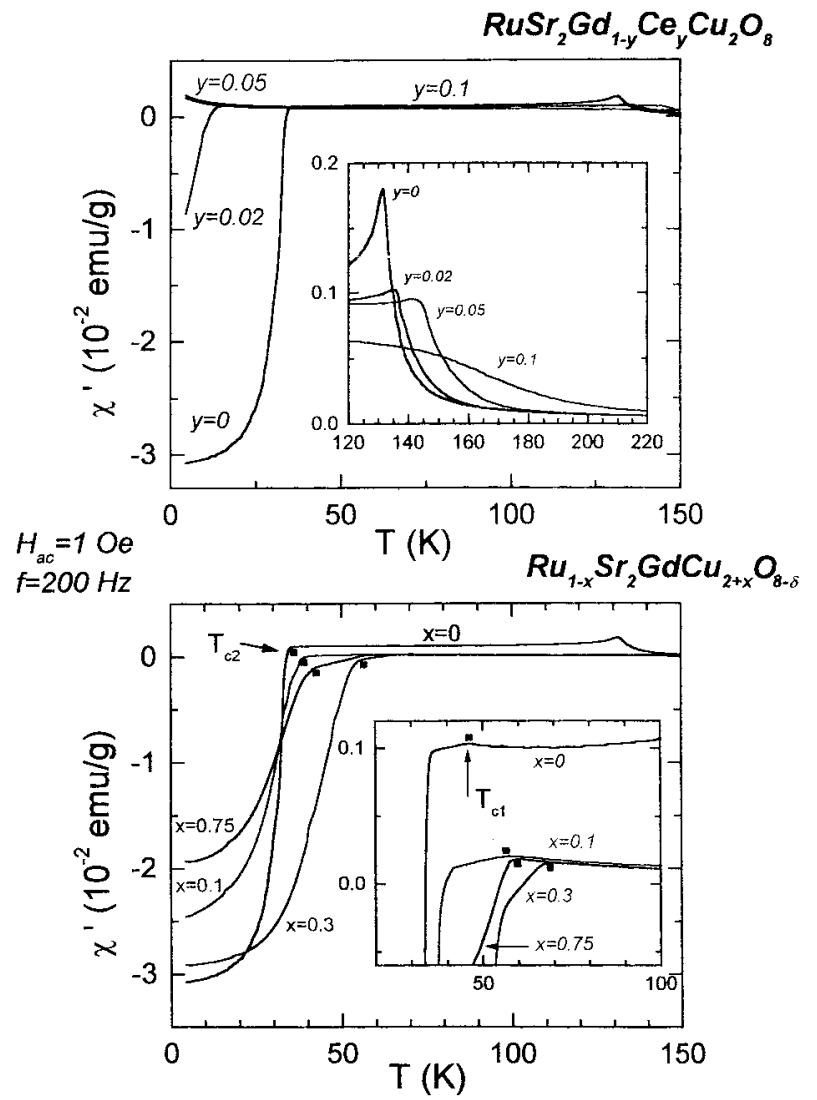

Fig. 3. ac susceptibility of the $\mathrm{RuSr}_{2} \mathrm{Gd}_{1-y} \mathrm{Ce}_{y} \mathrm{Cu}_{2} \mathrm{O}_{8}$ samples with $y=0-0.1$, insert: behavior near the AFM transition (top panel) and the $\mathrm{Ru}_{1-x} \mathrm{Sr}_{2} \mathrm{GdCu}_{2+x} \mathrm{O}_{8-d}$ samples with $x=0-0.75$, insert: details of the onset of the SC transition near $T_{\mathrm{c} 1}$ (bottom panel). 
to $62 \mathrm{~K}$ for the 0.75 sample (see Fig. 3 (bottom panel)). Although the field cooled and zero field cooled magnetization showed small irreversibility at $\sim 120 \mathrm{~K}$ and $100 \mathrm{~K}$, for $x=0.1$ and 0.2 , respectively, the low-temperature magnetization data did not reveal any extra magnetic contribution. Muon spin rotation $(\mu \mathrm{SR})$ experiments performed for the sample $x=0.1$ verified that the increase of the relaxation rate observed below $\sim 120 \mathrm{~K}$ should not be attributed to the bulk response of the material. For the $x=0.3$, 0.4 , and 0.75 compositions, no irreversibility of the magnetization in the normal state was observed. Thus, the $\mathrm{Cu}$ substituted samples do not show bulk magnetic transition above $T_{\mathrm{c} 1}$, probably because of dilution by $\mathrm{Cu}$ of the $\mathrm{Ru}$ magnetic sublattice. Highly sensitive $\mu \mathrm{SR}$ measurements at low temperatures revealed, however, new magnetic transitions appearing in the SC state below $T_{\mathrm{M}}=13,6$, and $2 \mathrm{~K}$, for $x=0.1$, 0.3 , and 0.4 , respectively [20]. The asymmetry parameters confirmed the bulk nature of these transitions. The undisturbed superconducting $\mathrm{CuO}_{2}$ planes appear to be responsible for the observed behavior. Similar coexisting AFM and $\mathrm{SC}$ order on $\mathrm{CuO}_{2}$ planes has been observed in other underdoped HTSC systems [21], $\mathrm{La}_{2-x} \mathrm{Sr}_{x} \mathrm{CuO}_{4}$ and $\mathrm{Y}_{1-x} \mathrm{Ca}_{x} \mathrm{Ba}_{2} \mathrm{Cu}_{3} \mathrm{O}_{6}$, for which the microscopic inhomogeneity of the charge distribution on the $\mathrm{CuO}_{2}$ planes and formation of the charge stripes have been proposed [22].

Resistivity measurements for pure Ru1212 showed metallic behavior that is enhanced by magnetic transition at $T_{\mathrm{m}}$ and the relatively broad SC transition with the onset at $T_{\mathrm{c} 1}$. The application of magnetic field smooths out transition at $T_{\mathrm{m}}$ and broadens the $\mathrm{SC}$ transition. For the $\mathrm{Cu}$ substituted samples, no resistive changes that could be associated with magnetic transitions in the normal state were detected in agreement with magnetic and $\mu \mathrm{SR}$ data. The SC transitions showed clear two steps: the first around $T_{\mathrm{c} 1}$ that do not depend on the weak magnetic fields and the second around $T_{\mathrm{c} 2}$ that is rapidly shifted to lower temperatures with application of weak magnetic fields of 100-1000 Oe. This behavior of resistivity is consistent with the quasi-two-dimensional character of the SC layers that are separated by nonsuperconducting regions. The resistivity of the Ce substituted samples $y=0-0.05$ remains metallic and the decrease of resistivity at $T_{\mathrm{m}}$ is enhanced. Consistent with magnetic measurements only the $y=0.02$ sample shows SC transition. Figure 4 (top panel) shows resistivity of the nonsuperconducting $y=0.1$ sample. Two transitions can be observed on cooling: the insulator/metal transition at $\sim 190 \mathrm{~K}$, and the metal/insulator transi-

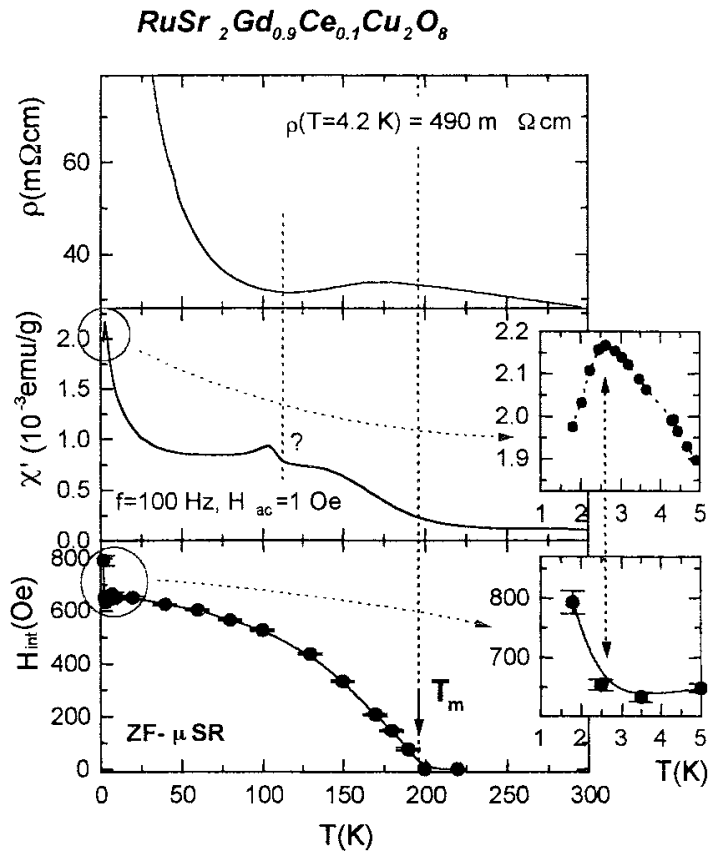

Fig. 4. Resistivity (top panel), susceptibility (middle panel), and the internal magnetic field from zero-field muon spin rotation experiments (bottom panel) of the nonsuperconducting $\mathrm{RuSr}_{2} \mathrm{Gd}_{0.9} \mathrm{Ce}_{0.1} \mathrm{Cu}_{2} \mathrm{O}_{8}$ sample. Inserts show details of the low temperature susceptibility and $\mu \mathrm{SR}$ data.

tion around $110 \mathrm{~K}$. Figure 4 (middle panel) and (bottom panel) of the susceptibility and zero-field $\mu \mathrm{SR}$ data, respectively, shows that the upper resistive transition is clearly associated with the magnetic transition at $T_{\mathrm{m}}$. The novel lower-temperature transition for this heavily underdoped nonsuperconducting composition seems to be associated with the susceptibility anomaly but no corresponding irregularity is seen for the $\mu \mathrm{SR}$ data. While more detailed $\mu \mathrm{SR}$ experiments are necessary to determine the origin of the lowertemperature transition, we can hypothesize at this time that it may be associated with the familiar AFM transition of the underdoped $\mathrm{CuO}_{2}$ planes.

The lowest temperature susceptibility and $\mu \mathrm{SR}$ data displayed on Fig. 4 (middle panel) and (bottom panel) show AFM transition of the Gd sublattice at $\sim 2.5 \mathrm{~K}$. Complimentary Mossbauer effect measurements [23] on ${ }^{155} \mathrm{Gd}$ for pure Ru1212 revealed that Gd moments are AFM ordered along the c-axis below $2.75 \mathrm{~K}$. Isomer shift measurements confirmed that $\mathrm{Gd}$ exists in a charge state $3+$. Similar measurements on ${ }^{99} \mathrm{Ru}$ showed that $\mathrm{Ru}$ orders AFM below $\sim 132 \mathrm{~K}$ as observed from susceptibility measurements and remain in this ordered state below the $\mathrm{SC}$ transition temperature. The isomer shift indicates 


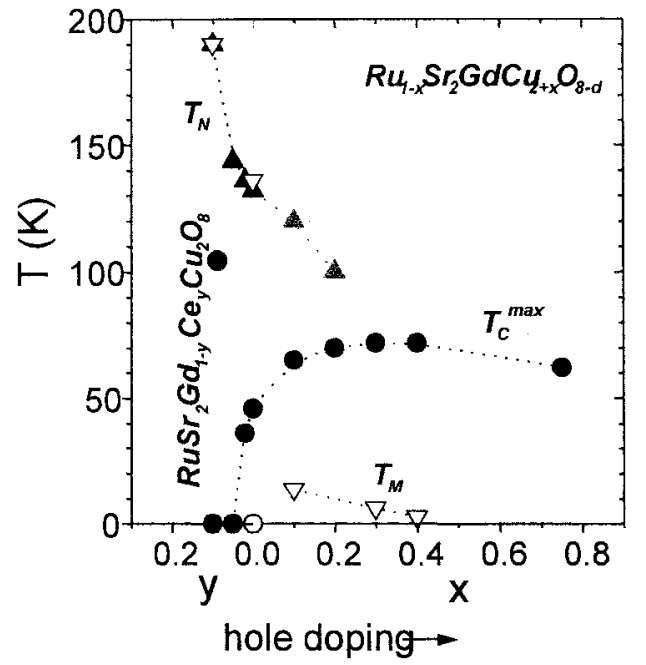

Fig. 5. Properties phase diagram for the $\mathrm{Ce}$ and $\mathrm{Cu}$ substituted $\mathrm{RuSr}_{2} \mathrm{GdCu}_{2} \mathrm{O}_{8}$ derived from susceptibility, magnetization, and resistive measurements of the SC (filled circles) and magnetic (filled triangles) transitions, and from muon spin rotation measurements of the magnetic transitions (open triangles). Filled circle for $y=0.1$ denotes new metal-insulator/magnetic transition.

an intermediate charge state between $4+$ and $5+$, $\mathrm{Ru}^{5-2 p}$, and the magnitude of magnetic hyperfine field points to a high spin configuration. While the XANES measurements of the $\mathrm{Cu} \mathrm{K}$-edge could not detect any difference of the hole doping between SC and non-SC samples for pure Ru1212, the measurements clearly showed enhanced doping of the $\mathrm{Cu}^{2+p}$ for the $\mathrm{Ru}_{1-x} \mathrm{Sr}_{2} \mathrm{GdCu}_{2+x} \mathrm{O}_{8-d}$ series. The charge carrier density at room temperature estimated from Hall effect measurements showed similar enhancement ranging from $1.7 \cdot 10^{27}$ to $3.9 \cdot 10^{27} 1 / \mathrm{m}^{3}$ for $x=0$ 0.4 , respectively, and a saturation for larger $x$. The thermopower measurements were consistent with the XANES and Hall effect data showing rapid drop of the thermopower coefficient between $x=0$ and 0.1 and more gradual decrease for larger $x$. Positive values in the normal state imply the underdoped nature of this superconductor for all $x$. Put together with structural, magnetic, and superconducting data, these measurements show conclusively quite uniform holedoping across the series of $\mathrm{Ce}$ and $\mathrm{Cu}$ substituted samples (see Ref. 20). Figure 5 shows the physical properties phase diagram derived from various experiments as a function of the utilized substitutions.

\section{CONCLUSIONS}

$\mathrm{RuSr}_{2} \mathrm{GdCu}_{2} \mathrm{O}_{8}$ is a unique Sr-based 123 compound with $\mathrm{Ru}$ completely replacing $\mathrm{Cu}$ in the chain region of the structure, resulting in the self-doped superconducting and magnetic material. The selfdoping is probably possible because of a similar energy levels for the $\mathrm{Ru}^{4+} / \mathrm{Ru}^{5+}$ and $\mathrm{Cu}^{2+} / \mathrm{Cu}^{3+}$ couples. By using chemical substitutions and controlling the oxygen pressure during synthesis and annealing, we have demonstrated that a wide range of the hole doping can be accomplished in Ru1212 compounds. With the Ce substitution for Gd, the AFM transition temperature of the $\mathrm{RuO}_{2}$ layers increases while the $\mathrm{SC}$ of the $\mathrm{CuO}_{2}$ planes rapidly disappears; for the most underdoped compositions achieved, a new magnetic transition emerges-probably the AFM state on the nonsuperconducting $\mathrm{CuO}_{2}$ planes. These both magnetic transitions are strongly coupled to the resistive properties. With the $\mathrm{Cu}$ substitution for $\mathrm{Ru}$, the coherent AFM state of the $\mathrm{RuO}_{2}$ layers is destroyed while the SC of the $\mathrm{CuO}_{2}$ planes is enhanced. New bulk magnetic state emerges at low temperatures, probably the AFM state on the superconducting $\mathrm{CuO}_{2}$ planes which is similar to those observed in other underdoped HTSC systems and associated with the charge stripe phases.

The inhibiting contribution of the polarized $\mathrm{Gd}^{3+}$ magnetic moments to the magnetization at low temperatures causes plausible absence of the Meissner state. Weak Ru ferromagnetism is augmented by the properties of the $\mathrm{Gd}^{3+}$ and significantly enhanced in the presence of the magnetic field. These results indicate constrained geometry of the superconducting phase in Ru1212. To unequivocally prove this behavior and measure the anisotropic properties, high quality single-crystals of pure and substituted compositions are indispensable. New hole-doping methods, which should not destroy the coherence of the $\mathrm{RuO}_{2}$ layers, are necessary to develop for probing the competition, and possibly the coexistence, of superconductivity and magnetism in these exciting materials.

\section{ACKNOWLEDGMENTS}

This work was supported by the U.S. National Science Foundation (DMR-0105398), and by the State of Illinois under HECA. We thank Dr I. Savic, Dr D. Herlach, and Dr A. Amato of PSI, Villigen, for their valuable assistance with the $\mu \mathrm{SR}$ experiments.

\section{REFERENCES}

1. J. G. Bednorz and K. A. Muller, Z. Phys. B 64, 189 (1986).

2. I. Felner, U. Asaf, Y. Levi, and O. Millo, Phys. Rev. B 55, R3374R3377 (1997). 
3. C. Bernhard, J. L. Tallon, Ch. Niedermayer, Th. Blasius, A. Golnik, E. Brucher, R. K. Kremer, D. R. Noakes, C. E. Stronach, and E. J. Ansaldo, Phys. Rev. B 59, 14099-14107 (1999).

4. V. L. Ginzburg, Sov. Phys. JETP 4, 153-160 (1957).

5. B. T. Matthias, H. Suhl, and E. Corenzwit, Phys. Rev. Lett. 1, 449-450 (1958).

6. M. B. Maple, Physica B 215,110-126 (1995).

7. Y. Dalichaouch, M. B. Maple, M. S. Torikachvili, and A. L. Giorgi, Phys. Rev. B 39, 2423-2431 (1989).

8. S. S. Saxena, P. Agarwal, K. Ahilan, F. M. Groshe, R. K. W. Haselwimmer, M. J. Steiner, E. Pugh, I. R. Walker, S. R. Julian, P. Monthoux, G. G. Lonzarich, A. Huxley, I Sheikin, D. Braithwaite, and J. Floquet, Nature 406, 587-592 (2000).

9. K. Nakamura, K. T. Park, A. J. Freeman, and J. D. Jorgensen, Phys. Rev. B 63, 024507 (2001).

10. J. W. Lynn, B. Keimer, C. Ulrich, C. Bernhard, and J. L. Tallon, Phys. Rev. B 61, R14964 (2000).

11. O. Chmaissem, J. D. Jorgensen, H. Shaked, P. Dollar, and J. L. Tallon, Phys. Rev. B 61, 6401 (2002).

12. J. D. Jorgensen, O. Chmaissem, H. Shaked, S. Short, P. W. Klamut, B. Dabrowski, and J. L. Tallon, Phys. Rev. B 63, 054440 (2001).

13. W. E. Pickett, R. Weht, and A. B. Shick, Phys. Rev. Lett. 83, 3713 (1999)
14. H. Shimahara, Phys. Rev. B 62, 3524 (2000).

15. C. W. Chu, Y. Y. Xue, R. L. Meng, J. Cmaidalka, L. M. Dezaneti, Y. S. Wang, B. Lorenz, and A. K. Heilman, cond-mat/ 9910056v3.

16. P. W. Klamut, B. Dabrowski, S. Kolesnik, and M. Maxwell, J. Mais, Phys. Rev. B 63, (2001) 224512.

17. B. Dabrowski, K. Rogacki, J. W. Koenitzer, K. R. Poeppelmeier, and J. D. Jorgensen, Physica C 277, 24 (1997).

18. P. W. Klamut, B. Dabrowski, S. Mini, M. Maxwell, S. Kolesnik, M. Mais, A. Shengelaya, R. Khasanov, I. Savic, H. Keller, T. Graber, J. Gebhardt, P. J. Viccaro, and Y. Xiao, Physica C 364365, 313 (2001).

19. P. W. Klamut, B. Dabrowski, J. Mais, and M. Maxwell, Physica $C$ 350, 24 (2001).

20. P. W. Klamut, B. Dabrowski, S. M. Mini, S. Kolesnik, M. Maxwell, J. Mais, A. Shengelaya, R. Khazanov, I. Savic, H. Keller, C. Sulkowski, D. Wlosewicz, M. Matusiak, A. Wisniewski, R. Puzniak, and I. Fita in Ruthenates and Rutheno-cuprate Materials: Theory and Experiment (SpringerVerlag, Berlin, 2002).

21. C. Niedermayer, C. Bernhard, T. Blasius, A. Golnik, A. Moodenbaugh, and J. I. Budnick, Phys. Rev. Lett. 80, 3843 (1998).

22. J. M. Tranquada, Phys. Rev. Lett. 78, 338 (1997).

23. R. Kruk, R. Kmiec, P. W. Klamut, B. Dabrowski, D. E. Brown, M. Maxwell, and C. W. Kimball, Physica C 370, 71 (2002). 